\title{
Unsteady Flow and Structural Behaviors of Centrifugal Pump under Cavitation Conditions
}

Denghao $\mathrm{Wu}^{1,2^{*}} \mathbb{0}$, Yun Ren ${ }^{2}$, Jiegang $\mathrm{Mou}^{1}$, Yunqing $\mathrm{Gu}^{1}$ and Lanfang Jiang ${ }^{2}$

\begin{abstract}
Cavitation has a significant effect on the flow fields and structural behaviors of a centrifugal pump. In this study, the unsteady flow and structural behaviors of a centrifugal pump are investigated numerically under different cavitation conditions. A strong two-way coupling fluid-structure interaction simulation is applied to obtain interior views of the effects of cavitating bubbles on the flow and structural dynamics of a pump. The renormalization-group $k-\varepsilon$ turbulence model and the Zwart-Gerbe-Belamri cavitation model are solved for the fluid side, while a transient structural dynamic analysis is employed for the structure side. The different cavitation states are mapped in the head-net positive suction head (H-NPSH) curves and flow field features inside the impeller are fully revealed. Results indicate that cavitating bubbles grow and expand rapidly with decreasing NPSH. In addition, the pressure fluctuations, both in the impeller and volute, are quantitatively analyzed and associated with the cavitation states. It is shown that influence of the cavitation on the flow field is critical, specifically in the super-cavitation state. The effect of cavitation on the unsteady radial force and blade loads is also discussed. The results indicate that the averaged radial force increased from $8.5 \mathrm{~N}$ to $54.4 \mathrm{~N}$ in the transition progress from an onset cavitation state to a super-cavitation state. Furthermore, the structural behaviors, including blade deformation, stress, and natural frequencies, corresponding to the cavitation states are discussed. A large volume of cavitating bubbles weakens the fluid forces on the blade and decreases the natural frequencies of the rotor system. This study could enhance the understanding of the effects of cavitation on pump flow and structural behaviors.
\end{abstract}

Keywords: Centrifugal pump, Cavitation, Unsteady flow, Structural behaviors, Fluid-structure interaction

\section{Introduction}

A centrifugal pump is a critical fluid transportation device and an important part of the petrochemical process loops. However, it is well-known that cavitation, which is inevitable in a centrifugal pump, can decrease the head-flow curve and increase noise and vibrations. In addition, blade surfaces have been eroded by the collapse of cavitation bubbles, which results in long-term damage [1]. In this study, particular attention will be given to the topic of unsteady flow and structural behaviors of a

\footnotetext{
*Correspondence: wudenghao@aliyun.com

${ }^{1}$ College of Mechanical Engineering, Zhejiang University of Technology, Hangzhou 310014, China

Full list of author information is available at the end of the article
}

centrifugal pump under cavitation conditions. In doing so, we will review previous studies.

In an actual system, cavitation flow in a centrifugal pump is so complex that, for instance, the transient characteristics of multiphase flow in pump and bubble dynamics are still not fully understood. Numerous studies have been conducted attempting to develop methods to study the flow behaviors of a centrifugal pump under cavitation conditions. Duplaa et al. [2] investigated the unsteady flow behaviors in cavitation during the pump startup. The unsteady pressures of the tongue, and suction and discharge areas, were measured and transient cavitating flow behaviors were identified. In addition, a particle image velocimetry technique and a high-speed camera image measuring and processing technology were used in attempts 
to better understand the transient flow structures of a centrifugal pump under cavitation conditions [3-5]. Hassan et al. [6] measured the vapor fraction through the turbopump using an X-ray technique and determined the spectra of dynamic pressure in the housing under different cavitation conditions. This provided a better understanding of the overall flow structures inside the pump inducer.

However, although experimental studies on the cavitation of pump have advanced significantly, there remain a number of issues that are still not fully solved. For instance, the measurement of the velocity of cavitating flow and vortex structures of shedding bubbles of cavitation are not yet feasible. To compensate for the shortage of experimental methods, computational fluid dynamics (CFD) methods were used, and its predictions of cavitation behaviors in centrifugal pumps revealed interesting results. During the simulation, the Reynolds-averaged Navier-Stokes (RANS) equations coupled with a homogeneous mixture of cavitation models were applied to obtain a clearer picture of transient cavitation flow structures and pressure pulsations in both the rotating part (impeller) and stationary part (volute or vane diffusor) [7-11]. In addition, a number of studies focused primarily on the structural behaviors of pumps under cavitation conditions. The vibration characteristics of centrifugal pumps under cavitation conditions were measured and the results indicated that the vibration amplitude increased significantly from non-cavitation to severe cavitation conditions $[12,13]$. d'Agostino et al. [14], Fu et al. [15], and Valentini et al. [16] studied the rotor-dynamic forces on cavitating pumps and the variations of rotor-dynamic forces corresponding to the cavitation states were reported.

In addition, to better understand the correlations between unsteady flow and the structural dynamic response in a centrifugal pump, a fluid-structure interaction (FSI) was conducted to investigate the interaction between fluids and structures in a pump. The FSI has the advantage that it describes the interaction of a deformable structure that is immersed in a fluid, where the fluid and structure motions influence each other. As a result, numerous engineering problems have been solved by FSI methods [17-20]. The one-way coupling method and two-way coupling method were used to investigate the deflection of an impeller, and the results indicated that the two-way coupling method yielded results that were close to actual measurements [21]. Pei et al. [22] expanded on this by investigating the unsteady flow-induced impeller oscillations for a single-blade pump by strong two-way coupling FSI simulations and non-contact deflection measurements under off-design conditions.
The above studies only cover a portion of the information regarding the effects of cavitation on flow instability and unstable structural behaviors in centrifugal pumps. In this study, more information of unsteady flow and structural behaviors of a centrifugal pump under cavitation conditions are investigated. A number of new features, for instance, the deformation, stress, and natural frequency data of a rotor system in a centrifugal pump under cavitation conditions, are analyzed by means of the FSI method.

\section{Physical Model and Simulation Method}

\subsection{Pump}

The pump is an ISO 80-50-250 standard centrifugal pump and, which is primarily used in petrochemical process areas. The sectional drawing of the pump is shown in Figure 1. The operational parameters are as follows: design flow rate $Q_{\mathrm{d}}=50 \mathrm{~m}^{3} / \mathrm{h}$, head $H_{\mathrm{d}}=80 \mathrm{~m}$, rotational speed $n=2900 \mathrm{r} / \mathrm{min}$, and $\mathrm{NPSH}=1.8 \mathrm{~m}$. The NPSH value, which is defined for describing cavitation states, is calculated based on the inlet total pressure, water vapor pressure, and density of the liquid at the ambient temperature. The geometrical parameters are as follows: impeller inlet diameter $D_{1}=80 \mathrm{~mm}$ impeller, outlet diameter $D_{2}=252 \mathrm{~mm}$, blade outlet height $b_{2}=6.5 \mathrm{~mm}$, and number of blades $Z=5$. The material properties of the impeller and shaft used in the structural analysis are presented in Table 1. The base material of both the impeller and shaft is DIN 1.4408 stainless steel.

\subsection{FSI Simulation Method}

There are two methods for the FSI simulation of a centrifugal pump: one-way coupling, and two-way coupling. The two-way coupling method is more accurate, however, it is time-consuming and requires greater computational resources. In this study, a two-way coupling method is applied to solve the FSI of a centrifugal pump under different cavitation conditions.

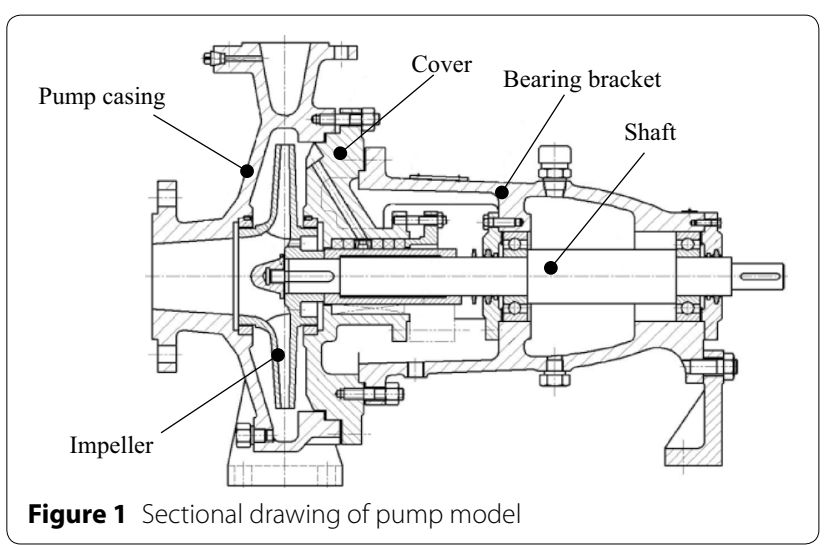


Table 1 Material properties of DIN 1.4408 stainless steel

\begin{tabular}{lll}
\hline Parameter & Symbol & Value \\
\hline Density $\left(\mathrm{kg} / \mathrm{m}^{3}\right)$ & $\rho_{\mathrm{m}}$ & 8000 \\
Modulus of elasticity $(\mathrm{Pa})$ & $E$ & $1.93 \times 10^{11}$ \\
Poisson's ratio & $\gamma$ & 0.29 \\
Tensile strength, ultimate $(\mathrm{MPa})$ & $\sigma_{\mathrm{b}}$ & 580 \\
Tensile strength, yield $(\mathrm{MPa})$ & $\sigma_{\mathrm{s}}$ & 290 \\
\hline
\end{tabular}

The strategy for the strong two-way coupling method is presented in Figure 2. In the one-time step simulation, the pressure forces from the fluid calculation are used as an input for the structural calculation, and the solution of the structural dynamics can be obtained under the effect of the pressure forces. The displacements from the structural calculation are then used as an input for the fluid calculation by interpolating to the fluid mesh that leads to its deformation.

For the three-dimensional fluid calculation, the unsteady Reynolds-averaged Navier-Stokes equations are solved by the CFD code ANSYS-CFX. The renormalization-group (RNG) $k-\varepsilon$ turbulence model with scalable wall functions is selected because of its high calculation accuracy. The turbulent kinetic energy equation for the RNG $k-\varepsilon$ turbulence model is defined in Ref. [23]:

$$
\begin{aligned}
& \frac{\partial(\rho k)}{\partial t}+\frac{\partial\left(\rho k u_{j}\right)}{\partial x_{j}}=\frac{\partial}{\partial x_{j}}\left(\alpha_{k} \mu_{\mathrm{e}} \frac{\partial k}{\partial x_{j}}\right)+\rho\left(P_{k}-\varepsilon\right), \\
& \frac{\partial(\rho \varepsilon)}{\partial t}+\frac{\partial\left(\rho \varepsilon u_{j}\right)}{\partial x_{j}}=\frac{\partial}{\partial x_{j}}\left(\alpha_{\varepsilon} \mu_{\mathrm{e}} \frac{\partial \varepsilon}{\partial x_{j}}\right)+\rho \frac{\varepsilon}{k}\left(C_{1 \varepsilon}^{*} P_{k}-C_{2 \varepsilon} \varepsilon\right),
\end{aligned}
$$

where $\quad C_{1 \varepsilon}^{*}=C_{1 \varepsilon}-\frac{\eta\left(1-\eta / \eta_{0}\right)}{1+\beta \eta^{3}}, \quad \eta=\left(2 E_{i j} \cdot E_{i j}\right)^{1 / 2} \frac{k}{\varepsilon}$, $E_{i j}=\frac{1}{2}\left(\frac{\partial u_{i}}{\partial x_{j}}+\frac{\partial u_{j}}{\partial x_{i}}\right), \mu_{\mathrm{e}}$ is the effective viscosity coefficient, $E_{i j}$ is the time-averaged strain rate, $P_{k}$ is the turbulent kinetic energy, $\alpha_{k}=\alpha_{\varepsilon}=1.39, C_{1 \varepsilon}=1.42, C_{2 \varepsilon}=1.68$, $\eta_{0}=4.377$, and $\beta=0.012$.

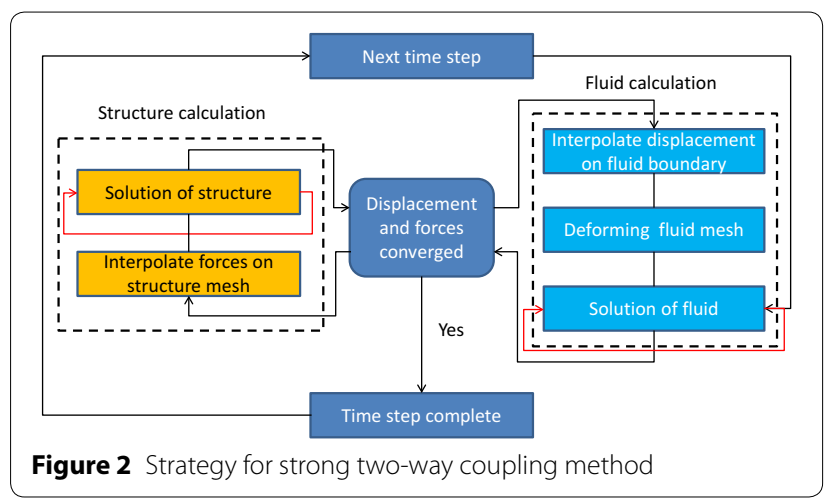

The total inlet pressure and outlet mass flow rate are set as the boundary conditions. The cavitation state is changed by adjusting the inlet pressure. For the cavitation calculation, the Zwart-Gerber-Belamri model, which is based on the Rayleigh-Plesset equation, was chosen. More detailed information of the numerical settings is presented in Table 2. For both the cavitation and non-cavitation conditions, the convergence residual of calculation is $1.0 \times 10^{-5}$. For the transient simulation, the time step is set as $1.724 \times 10^{-4} \mathrm{~s}$, which is $1 / 120$ of the rational period $T$.

The computational fluid domains are meshed in structured grids by means of ANSYS-ICEM. The grid independence is checked for simulations at the design flow rate. Five different grid numbers are created and calculated in the CFD code under two different cavitation conditions $(\mathrm{NPSH}=20 \mathrm{~m}$ and $\mathrm{NPSH}=2.58 \mathrm{~m})$. As can be seen in Figure 3, the simulated head remains constant when the grid number reaches approximately 1.1 million (Grid 3). Therefore, considering both the accuracy and time for the calculations, Grid 3 is selected for the simulations. The solid domains are meshed in unstructured grids using ANSYS Meshing, and total grid number is approximately 0.45 million elements. The meshes of the fluid and solid domains are shown in Figures 4 and 5, respectively. In addition, a number of monitoring points are defined in different regions of the pump in an attempt to collect unsteady information of the inner flow field during the simulation. The monitoring points in the impeller and volute are shown in Figure 6 . The pressure information is collected and calculated into dimensionless values for the analysis. The equation of dimensionless pressure is:

$$
c_{\mathrm{p}}=\frac{p-\bar{p}}{0.5 \rho u_{2}^{2}},
$$

where $p$ is the transient pressure, $\bar{p}$ is the mean pressure, $\rho$ is the density of the liquid, and $u_{2}$ is the circumferential velocity at the impeller outlet.

Table 2 Fluid simulation settings

\begin{tabular}{ll}
\hline Parameter & Value \\
\hline Turbulence model & RNG $k-\varepsilon$ \\
Cavitation model & $\begin{array}{c}\text { Zwart-Ger- } \\
\text { ber-Belamri }\end{array}$ \\
& model \\
Evaporation coefficient & 50 \\
Condensation coefficient & 0.01 \\
Temperature (K) & 298 \\
Vapor pressure $(\mathrm{Pa})$ & 3574 \\
Inlet boundary & Total pressure \\
Outlet boundary & Mass flow rate \\
Roughness of wall $(\mathrm{mm})$ & 0.03 \\
\hline
\end{tabular}




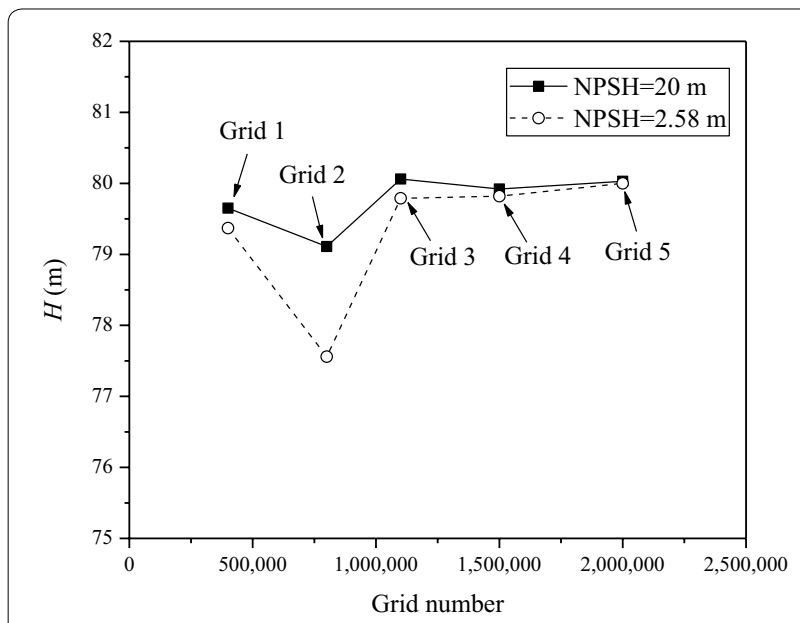

Figure 3 Mesh validation under two cavitation conditions

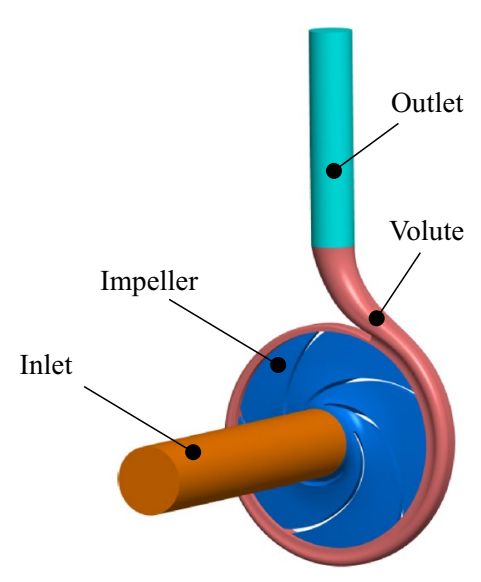

a Fluid domains

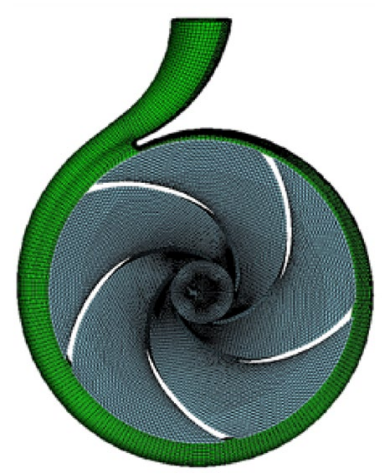

b Meshes for fluid calculation

Figure 4 Fluid domains and meshes

\section{Simulation Validation and Analysis}

\subsection{Test Setup and Validation}

To validate the simulation results, three typical flow rates, $0.8 Q_{\mathrm{d}}, 1.0 Q_{\mathrm{d}}$, and $1.2 Q_{\mathrm{d}}$, are measured in the test

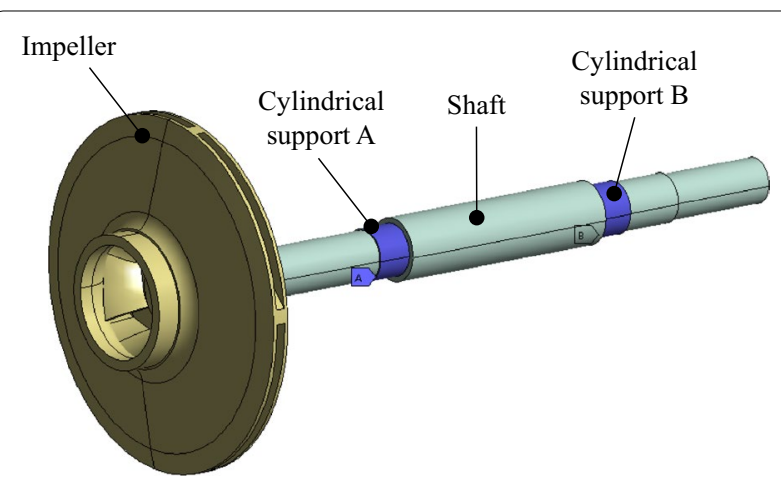

a Solid domains

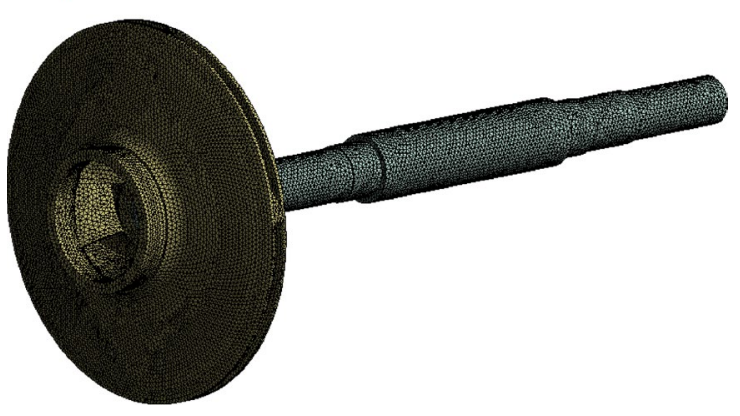

b Meshes for structure calculation

Figure 5 Solid domains and meshes

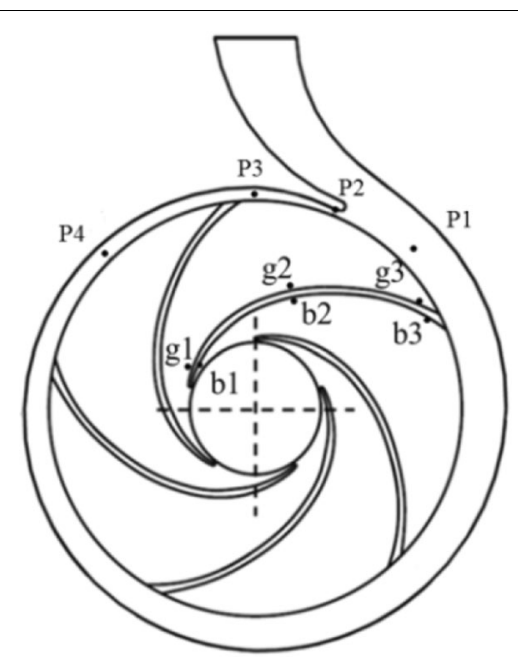

Figure 6 Pump monitoring points

rig. The test rig is shown in Figure 7. It is a closed-loop system, and was designed according to ISO 9906. To ensure a high experimental accuracy, the following maximum uncertainties are established for the measured magnitudes: 1 ) static pressure: $\pm 1.5 \% ; 2$ ) flow rate: $\pm 3 \%$; 


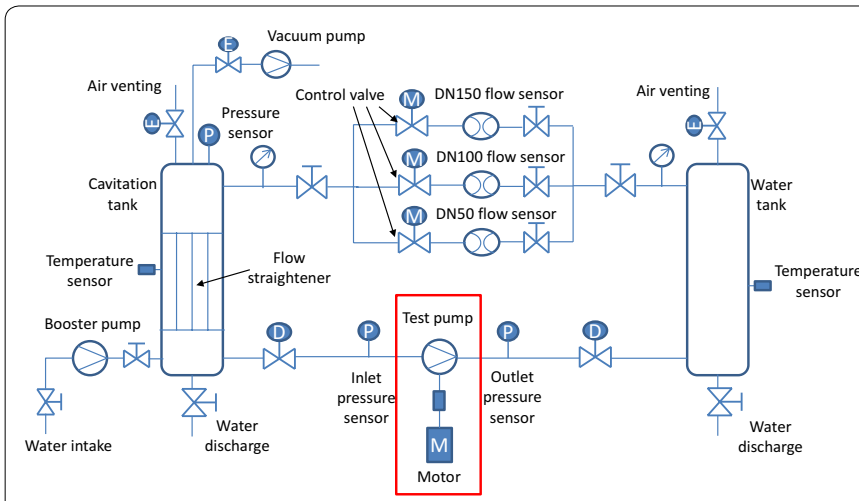

a Closed test rig for NPSH measurement

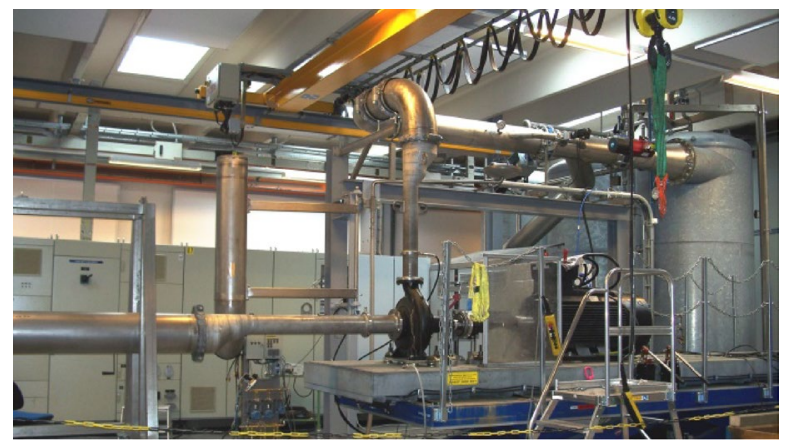

b Test setup

Figure 7 Test rig and facilities for pump NPSH measurements

3) rotational speed: $\pm 0.5 \%$; and 4 ) shaft power: $\pm 3 \%$. To keep the flow rate constant in the cavitation test, the inlet pressure is gradually decreased by adjusting the vacuum pump, and the flow, head, and inlet pressure are measured. The NPSH is progressively reduced until the decreasing total head at constant flow rate reaches $3 \%$. The cavitation data of the experiment and the simulation are shown in Figure 8. As the NPSH decreases but is not fully cavitated, the head remains approximately constant and, when the NPSH decreases to a certain value, the head decreases rapidly. This critical cavitation value is defined when the pump head reaches $3 \%$ of the non-cavitation head. The critical point indicates that the pump is fully cavitated and significant energy loss has occurred. The simulated values are in good agreement with the experimental results, and the error is within 3\%, which indicates that the calculation model is accurate and feasible. The NPSH is defined as follows:

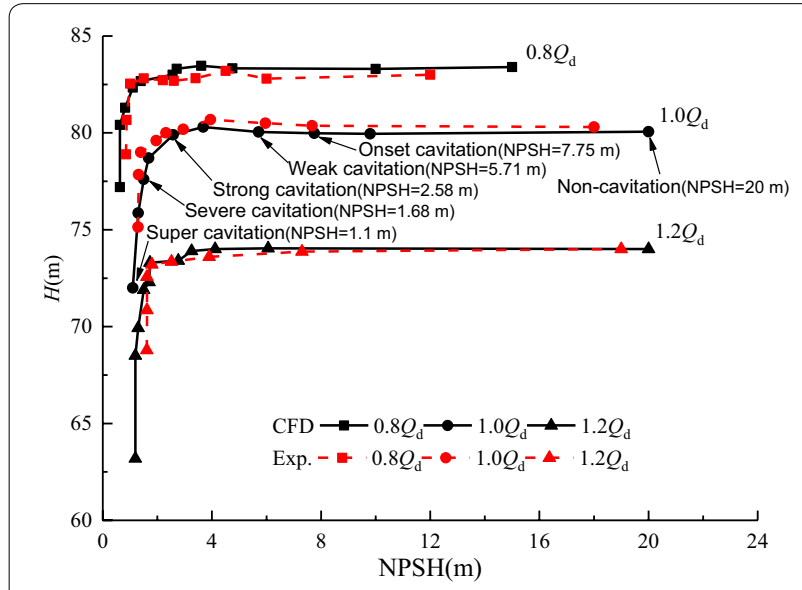

Figure 8 H-NPSH curve under different flow rate conditions

$$
\mathrm{NPSH}=\frac{P_{\text {total }}^{\text {inlet }}-P_{\mathrm{v}}}{\rho^{\prime} g}
$$

where $P_{\text {total }}^{\text {inlet }}$ is the total pressure of inlet, $\mathrm{Pa} ; P_{\mathrm{v}}$ is the vapor pressure of water, $\mathrm{Pa}$; and $\rho^{\prime}$ is the local density of the mixture, $\mathrm{kg} / \mathrm{m}^{3}$.

\subsection{Cavitation States Analysis}

To better understand the flow and structural behaviors of a pump under cavitation conditions, all the results are evaluated at the design flow rate. Figure 9 shows the distribution of vapor volume and flow streamlines in the impeller corresponding to the change of cavitation at the design flow rate. In order to monitor the onset of cavitation in the impeller, the isosurface distribution of $5 \%$ vapor volume faction is defined. It can be seen that the bubble volume gradually increases from the onset cavitation state to the super-cavitation state. The bubbles first appear at the inlet of the impeller, because the impeller inlet pressure is initially lower than the vaporization pressure. With the decrease in the inlet pressure, the bubble volume gradually expands from the impeller inlet to outlet. It is observed that the bubble volume increased rapidly under the severe cavitation condition and, finally, the bulk of the flow channels are occupied by the cavitation bubbles in the super-cavitation state.

As can be seen in Figure 9, the impeller is in an onset cavitation state when the NPSH $=7.75 \mathrm{~m}$, where the bubbles are small and located close to the leading edge of the blade on the suction side. When the NPSH $=5.71 \mathrm{~m}$, the bubble volume is small and increases slowly close to the suction side of the blade, which is defined as a weak cavitation state. When the NPSH $=2.58 \mathrm{~m}$, the bubbles are generated on both the suction and pressure sides of the 

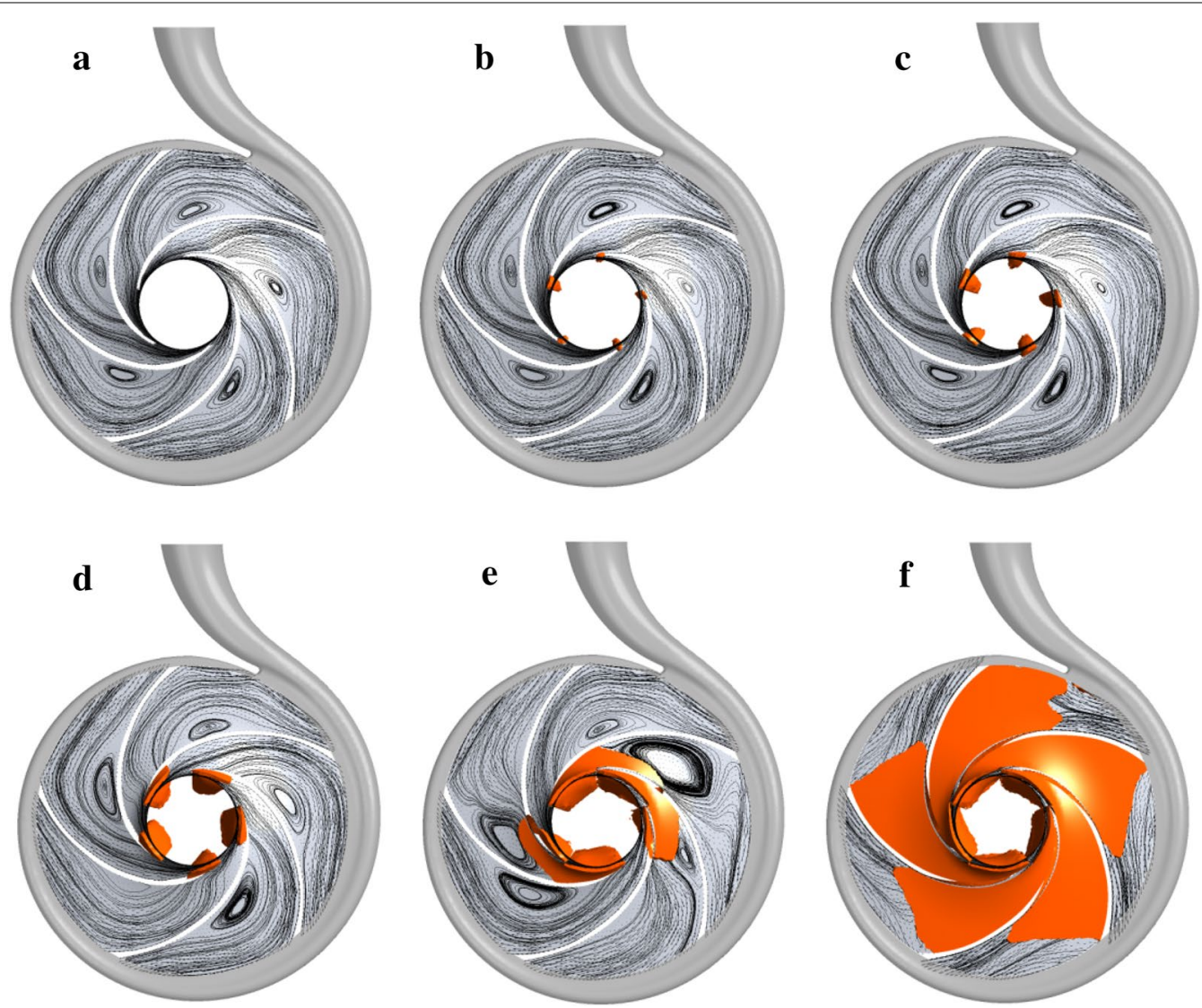

Figure 9 Vapor volume fraction and streamlines at mid-span of impeller (1.0Q): (a) Non-cavitation; (b) NPSH=7.75 m; (c) NPSH=5.71 m; (d) $\mathrm{NPSH}=2.58 \mathrm{~m}$; (e) $\mathrm{NPSH}=1.68 \mathrm{~m}$; and (f) $\mathrm{NPSH}=1.1 \mathrm{~m}$

blade. The impeller inlet is blocked under the strong cavitation state. When the NPSH $=1.68 \mathrm{~m}$, one quarter of the impeller flow channels is occupied by the cavitating bubbles under the severe cavitation state. The severe cavitation state indicates that the bubble volume grows rapidly, resulting in significant flow separation and energy loss and, therefore, poorer hydraulic performance. Finally, when the NPSH=1.1 m, it is defined as a super-cavitation state, and it can be seen in Figure 9(f) that the flow channels are completely blocked by the cavitating bubbles and the pump head has significantly decreased.

By analyzing the streamlines of the impeller in Figure 9, it can be seen that, when cavitation transitions from a non-cavitation state to a severe cavitation state, the location of the vortex moves from the pressure side of the blade to the suction side of the blade. This is because the impeller flow channels are almost blocked by the cavitating bubbles, and the flow separation on the pressure side of the blade is avoided by decreasing the flow velocity. Simultaneously, the backflow from the volute results in the flow separation and recirculation on the suction side of the blade. The recirculation zone increases with the significant kinetic energy loss corresponding to the decrease in NPSH. Finally, it results in the head decease of the centrifugal pump.

\section{Flow Behaviors Analysis under Cavitation Conditions}

4.1 Pressure Fluctuations of Impeller

Figure 10 shows the maximum amplitude of the dimensionless pressure fluctuations at the different monitoring points on the suction and pressure sides of the blade under different cavitation conditions. In Figure 10, it can be seen that: 1) the amplitude of the pressure fluctuation close to the suction side is significantly smaller than that of the pressure side at the same radius. At the same time, as the blade radius increases, from the blade inlet to the outlet, the pressure fluctuation amplitude gradually increases. In the non-cavitation state, the amplitude of the pressure fluctuation at monitoring point b1 is approximately 0.0012 , and the amplitude of the pressure fluctuation increased to 0.007 at monitoring point b3, which is located close to the outlet of the blade on the suction side. Comparing monitoring points g1 and g3, 


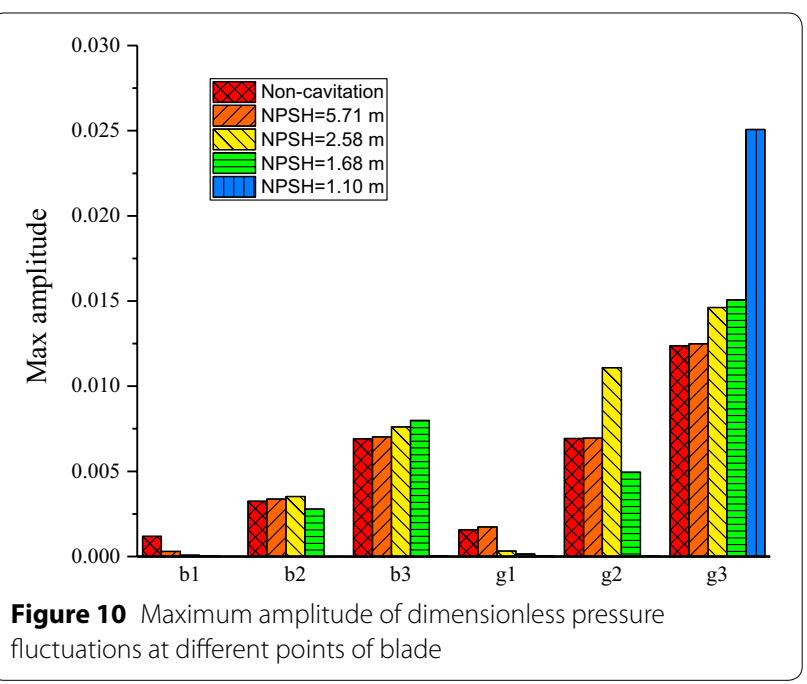

the corresponding pressure fluctuation amplitudes are 0.0016 and 0.012 , respectively. 2) When the NPSH $=5.71$ $\mathrm{m}$, the impeller is in a weak cavitation state, and only the blade suction side close to the impeller inlet (location of b1) is cavitating. Therefore, the pressure fluctuations from the flow domains are isolated by the cavitating bubbles, which results in the amplitude of the pressure fluctuation inside the bubble zone significantly decreasing. However, the amplitude of the pressure fluctuation outside the bubble zone is essentially at the same level as that of the non-cavitation state. 3) When the NPSH $=2.58 \mathrm{~m}$, the impeller is in a strong cavitation state, where cavitation expands to the locations of b1 and g1 and causes the amplitude of the pressure fluctuation to decrease significantly inside the bubble zone. While the pressure pulsation amplitude of the other monitoring points increased compared to the non-cavitation state, the pressure pulsation amplitude at monitoring points g2 and g3 increased significantly because a part of the impeller inlet is blocked by the cavitating bubbles and that enhances the flow velocity, which exacerbates the flow separation in the impeller flow path, resulting in a greater vortex and higher pressure fluctuations. These observations were made based on Figure 9. 4) When the $\mathrm{NPSH}=1.68 \mathrm{~m}$, the impeller is in a severe cavitation state, no fluctuation is observed at monitoring points b1 and g1, and the pressure fluctuation amplitudes of b2 and g2 are decreased because of the effect of the bubbles. However, the amplitude of pressure fluctuations at b3 and g3 actually increased. 5) When the NPSH $=1.1 \mathrm{~m}$, the impeller is in a super-cavitation state and the flow channels are fully occupied by the cavitating bubbles. Therefore, no fluctuation occurs at monitoring points b1, b2, g1, and $\mathrm{g} 2$ because they are located inside the bubble zone.
However, the flow outside the bubble zone is significantly affected by the large bubbles, which causes the flow pressure to vary greatly and, therefore, causes the amplitude of the pressure fluctuation at g3 to increase rapidly. The influence of cavitation on the pressure fluctuations in the impeller can be summarized as follows. Because of the isolation effects of the cavitating bubbles, the bubbles weaken the amplitude of the pressure fluctuation inside the bubble zone. However, it is the blockage and collapse of bubbles that enhances the vortex in the impeller and further increases the amplitude of the pressure fluctuation in the non-cavitation zone at, for instance, monitoring point $\mathrm{g} 3$.

\subsection{Pressure Fluctuations of Volute}

Figure 11 shows the time-domain diagram of the pressure fluctuations at monitoring points $\mathrm{P} 1-\mathrm{P} 4$ in the pump volute under different cavitation conditions at the design flow rate. Note that the pressure fluctuation amplitude is high at points P2 and P3 close to the volute tongue, and the pressure fluctuation is low at points P1 and $\mathrm{P} 4$ at a distance from the tongue, because the bladetongue interaction is stronger close to the volute tongue. It is observed that the pressure is fluctuating periodically, and the number of waves is consistent with the number of blades. It can also be seen that the phases of the peaks at each monitoring point in the volute are different. This is because the time of a blade passing the different monitoring points is not the same. From Figures 11 and 12, it is found that: 1) in the transition from the non-cavitation state to the severe cavitation state, the pressure fluctuations at the monitoring points in the volute are essentially the same, and the maximum pressure pulsation amplitude remains constant. 2) The pressure fluctuations at monitoring points $\mathrm{P} 2, \mathrm{P} 3$, and P4 are significantly increased when the impeller is in a super-cavitation state, and the corresponding maximum amplitudes increase to $0.17,0.29$, and 0.24 respectively. This indicates that, when the cavitation does not enter the super-cavitation state, it has little effect on the pressure fluctuation in the volute, and when the cavitation develops to a super-cavitation state, the large area of the cavitating bubbles changes the distribution of the pressure pulsation in the volute, and affects the flow characteristics of the volute that further enlarges the amplitude of the pressure fluctuations.

Figure 13 shows the pressure changes in the volute at different times in the super-cavitation state. It can be seen that the pressure changes at P2, P3, and P4 (red dashed box) close to the tongue area are more intense because of the effect of the large area of bubbles at different times, 


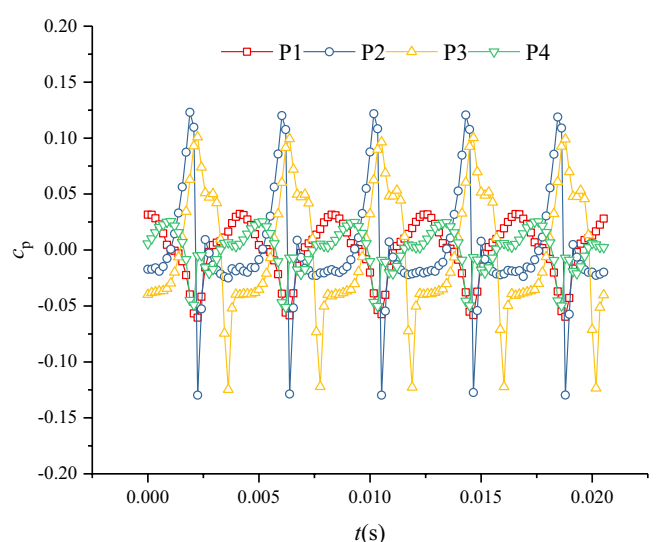

a Non-cavitation

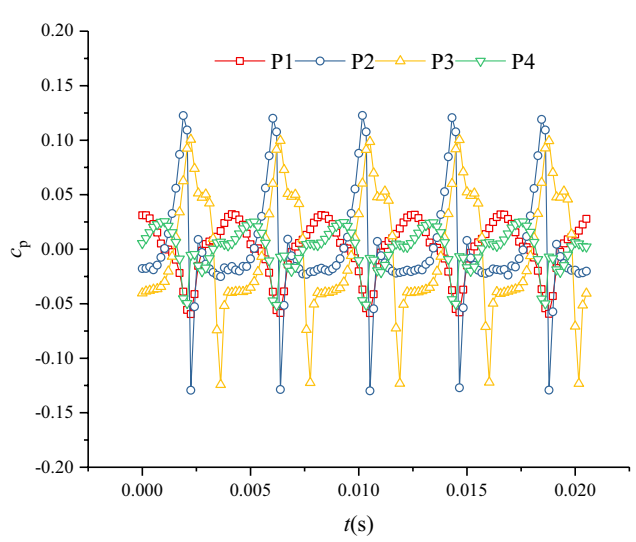

b $\mathrm{NPSH}=5.71 \mathrm{~m}$

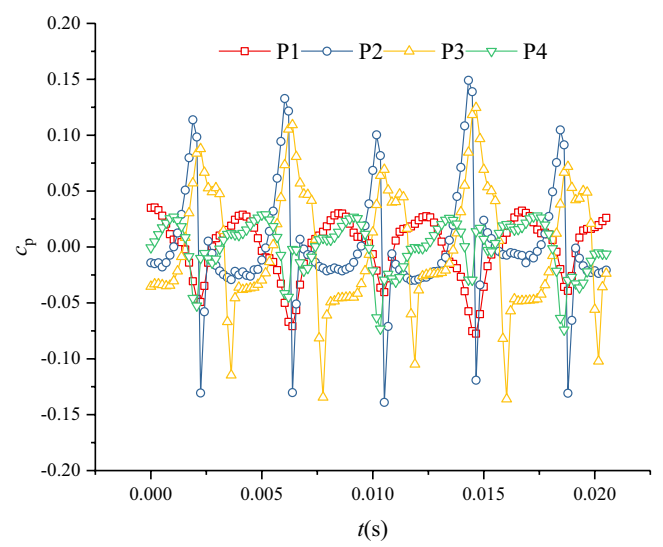

c $\mathrm{NPSH}=2.58 \mathrm{~m}$

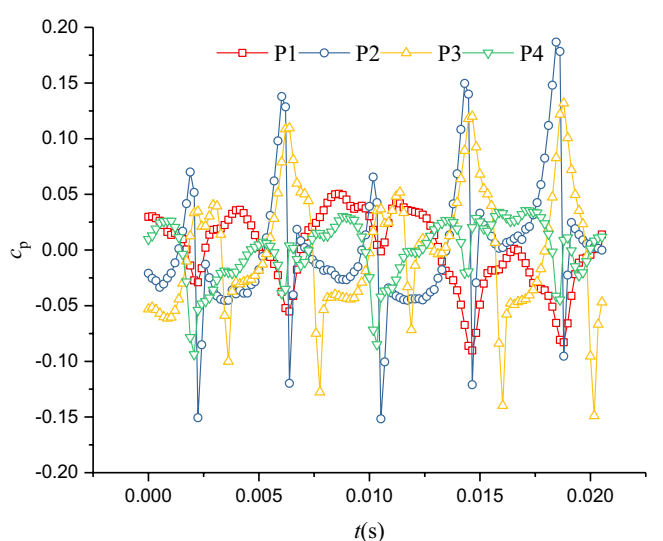

d $\mathrm{NPSH}=1.68 \mathrm{~m}$

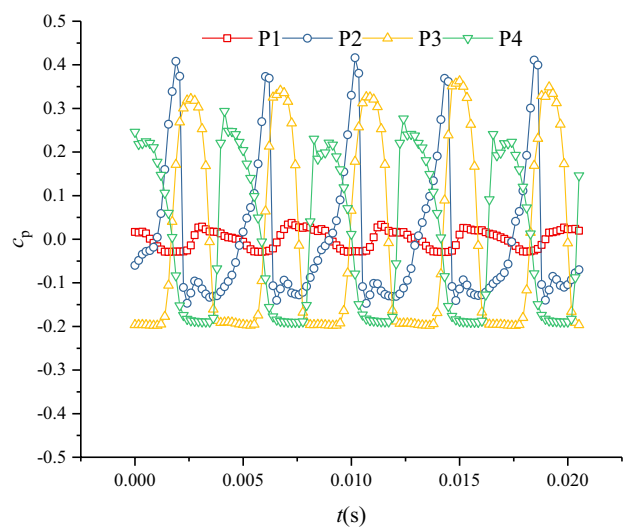

e $\mathrm{NPSH}=1.1 \mathrm{~m}$

Figure 11 Dimensionless pressure fluctuations at different points of volute

where the high- and low-pressure regions are alternating and changing significantly. However, the pressure at P1, at a distance from the tongue (blue dashed box), is relatively stable and remains low. In general, it is shown that the amplitude of the pressure oscillations is affected by bubble size and the volute tongue.

\subsection{Unsteady Blade Loads Analysis}

Figure 14 shows the radial force distribution of the impeller under different cavitation conditions at the design flow rate. It can be seen that the transient radial force of the impeller is fluctuating periodically in the non-cavitation state and the weak cavitation state. However, the 


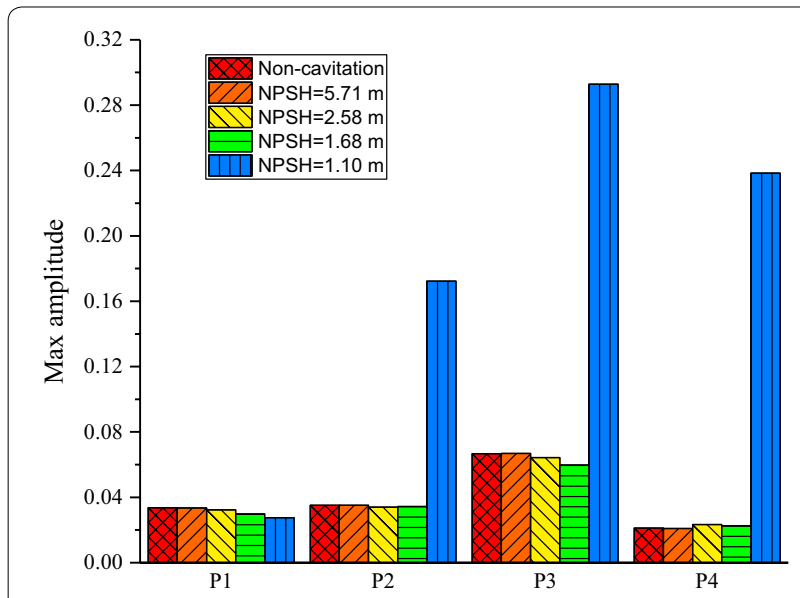

Figure 12 Max amplitude of dimensionless pressure fluctuations at different points of volute periodicity of the radial force fluctuation is weakened after the impeller enters the strong cavitation state, and its amplitude is significantly increased. This indicates that the development of cavitation affects the pressure loads on the blades. Furthermore, it can be seen from Figure 15 that the average radial force in a rotation cycle remains low, approximately $8.5 \mathrm{~N}$, at the beginning of cavitation. This is because, in this period, the bubbles only appear at the impeller inlet, and the flow velocity and pressure of the fluid in the whole flow path are less affected by cavitation, resulting in the radial force being at approximately the same level as in the non-cavitation state. When the cavitation develops to a severe cavitation state or supercavitation state, a significant amount of cavitating bubbles are generated and have a significant effect on the impeller flow. This results in the pressure distributions of the volute becoming non-uniform and the symmetrical flow state in the impeller channels being destroyed.

$$
\begin{array}{ll}
\text { Pressure } & {[\mathrm{Pa}]}
\end{array}
$$

a

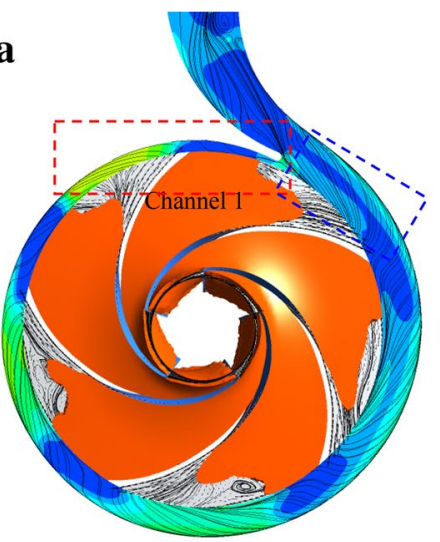

d

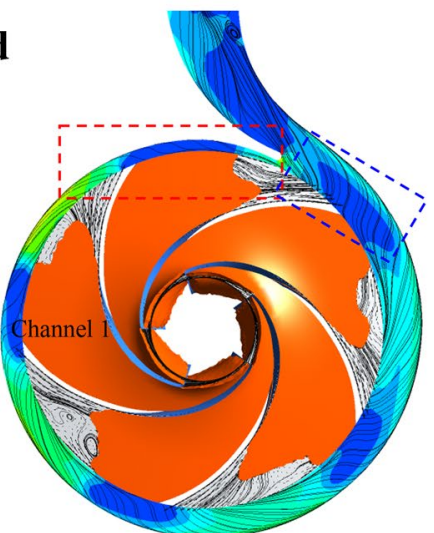

b

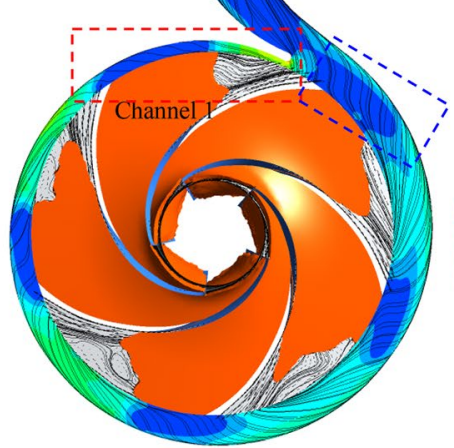

e

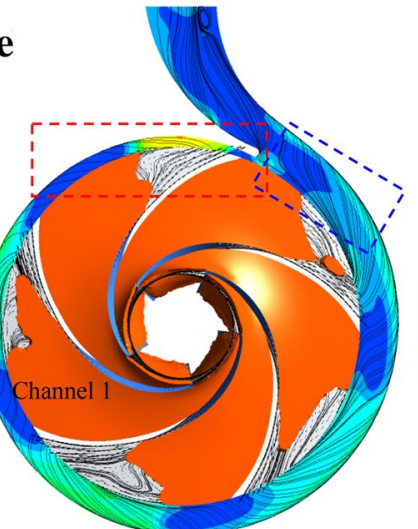

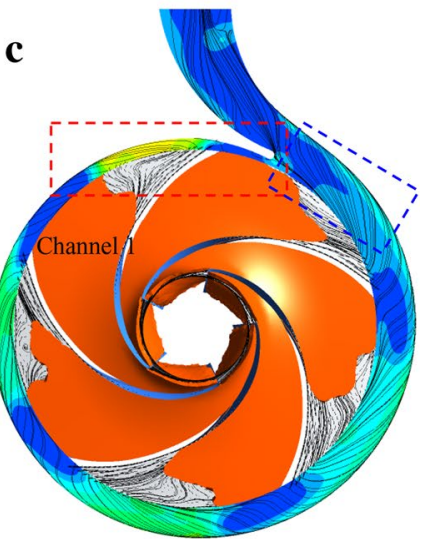

f

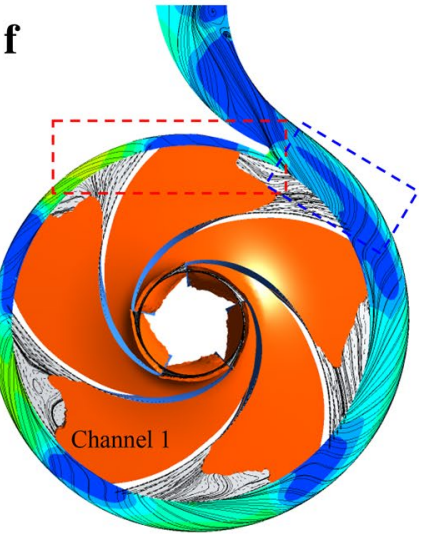

Figure 13 Transient pressure and vapor volume distribution under NPSH $=1.1 \mathrm{~m}$ : (a) $t_{0} ;(\mathbf{b}) t_{1}=t_{0}+0.00172 \mathrm{~s} ;(\mathbf{c}) t_{2}=t_{0}+0.00344 \mathrm{~s} ;(\mathbf{d})$ $t_{3}=t_{0}+0.00516 \mathrm{~s} ;(\mathbf{e}) t_{4}=t_{0}+0.00688 \mathrm{~s} ;$ and $(\mathbf{f}) t_{5}=t_{0}+0.00860 \mathrm{~s}$ 


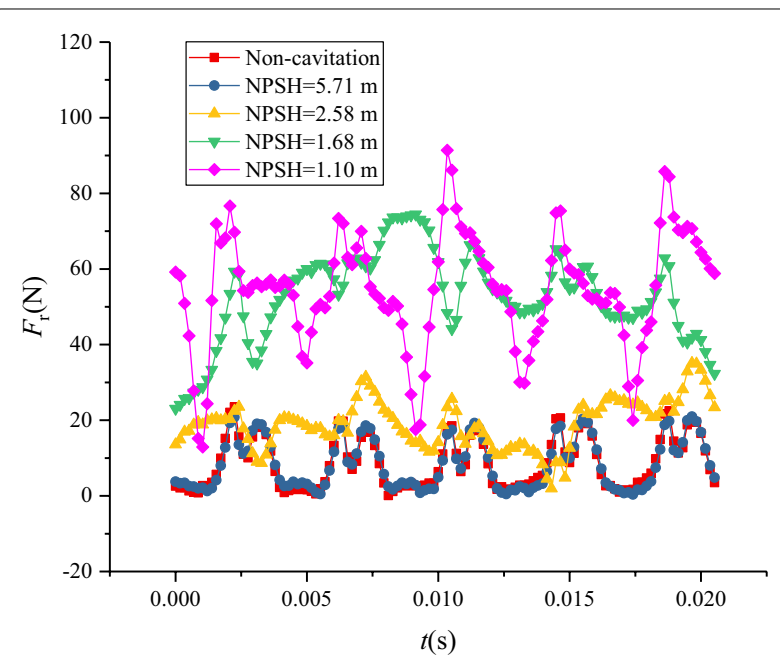

Figure 14 Unsteady radial force of impeller under different cavitation states

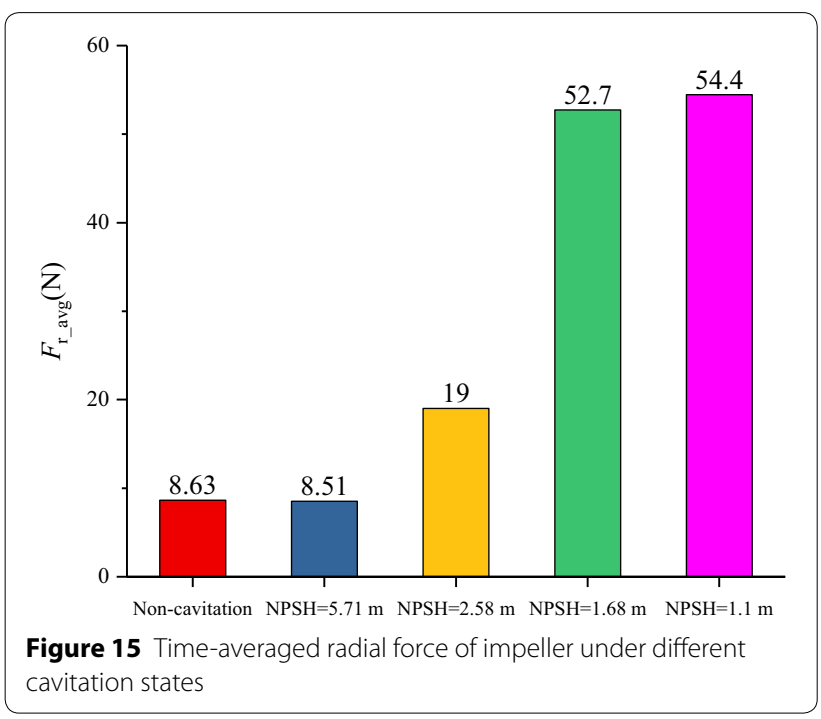

Therefore, a greater radial force is produced, and it increases significantly until the maximum value of $54.4 \mathrm{~N}$ is attained in the super-cavitation state.

In order to better understand the variations in the impeller radial force, a pressure loads analysis on a blade is conducted The results are shown in Figure 16, where $L$ is a dimensionless length of a blade from the leading edge (LE) to the trailing edge (TE), $L=0$ is the LE, and $L=1$ is the TE. Figure 16 shows that the blade load curves are similar from a non-cavitation state to a strong cavitation state $(\mathrm{NPSH}=2.58 \mathrm{~m})$. In the case of severe cavitation $(\mathrm{NPSH}=1.68 \mathrm{~m})$, the blade loads of the pressure side decreased and became similar to the suction side close to the LE. However, for a super-cavitation state
$(\mathrm{NPSH}=1.1 \mathrm{~m})$, the blade loads change significantly because of the effect of the large cavitating bubbles. From the LE to the middle of blade, the pressure loads on the suction and pressure sides are similar (black dashed box). It is clear that the blade loads on the pressure side increase rapidly from the middle of the blade to the TE, with a maximum load difference of approximately 0.4 . This is double the load of the other cavitation states. It also can be observed that the blade load curves break at the TE because of the effects of the bubbles in a supercavitation state.

\section{Structural Behaviors Analysis under Cavitation Conditions}

\subsection{Deformation and Stress Analysis of Blade}

Figure 17 shows the deformation of four different blade profiles under different cavitation states, where $\mathrm{PH}$ represents the intersection of the blade pressure surface with the hub, PS represents the intersection of the blade pressure surface with the shroud, $\mathrm{SH}$ represents the intersection of the blade suction surface with the hub, and SS represents the intersection of the blade suction surface with the shroud. It can be seen that in the transition progress from the non-cavitation state to the severe cavitation state, the blade deformation is approximately constant, with a maximum value of approximately 0.21 $\mathrm{mm}$. When the impeller enters the super-cavitation state, it is clear that the deformation of blade decreases slightly from the LE to the middle of the blade. However, from middle of the blade to the TE, the deformation decreases significantly, with a maximum value of $0.19 \mathrm{~mm}$. It is also observed that the deformation gradually increases from the blade inlet to the outlet. From the analysis of the blade deformation and blade load curves, it can be seen that the deformation distribution tendency is consistent with the blade load distribution shown in Figure 16. For example, before the super-cavitation state, both the deformation and blade loads are constant from the blade inlet to the outlet, regardless of the effect of cavitation. In the super-cavitation state, both the deformation and blade loads are going decrease, which is in good agreement with the law that states for the same loading surface, the greater the load, the greater the deformation. Therefore, it is shown that the development of cavitation changes the load distribution of the blade surface, which in turn changes the deformation of the blade surface.

Figure 18 shows the stress distribution of different blade profiles under different cavitation states. It can be seen that: 1) The stress on the four blade profiles gradually decreases with the development of cavitation. Before the super-cavitation state, the stress decrease is small and the overall distribution tendencies are approximately the same. However, in the super-cavitation state, the stress 


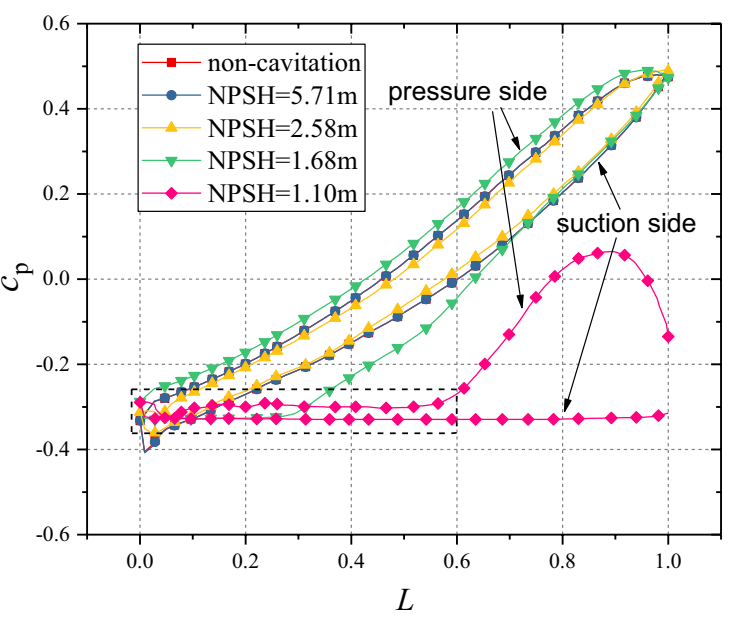

a Span0.1 (near hub)

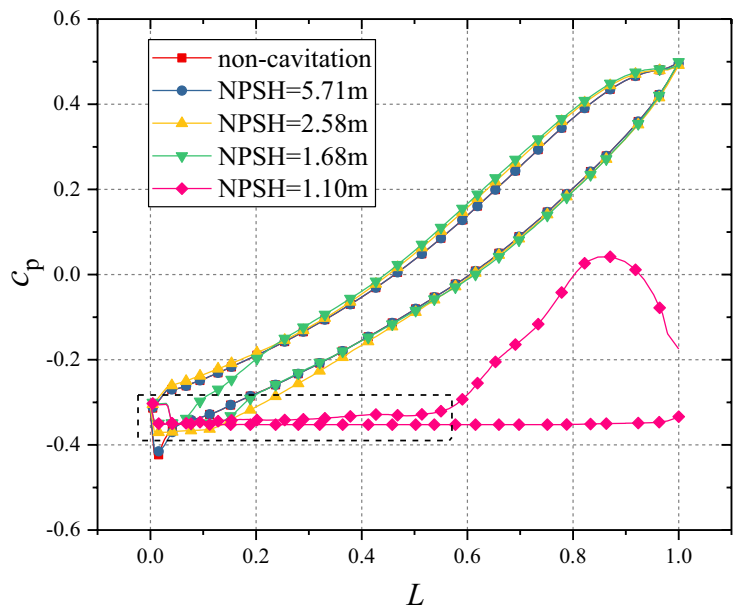

b Span0.5 (middle)

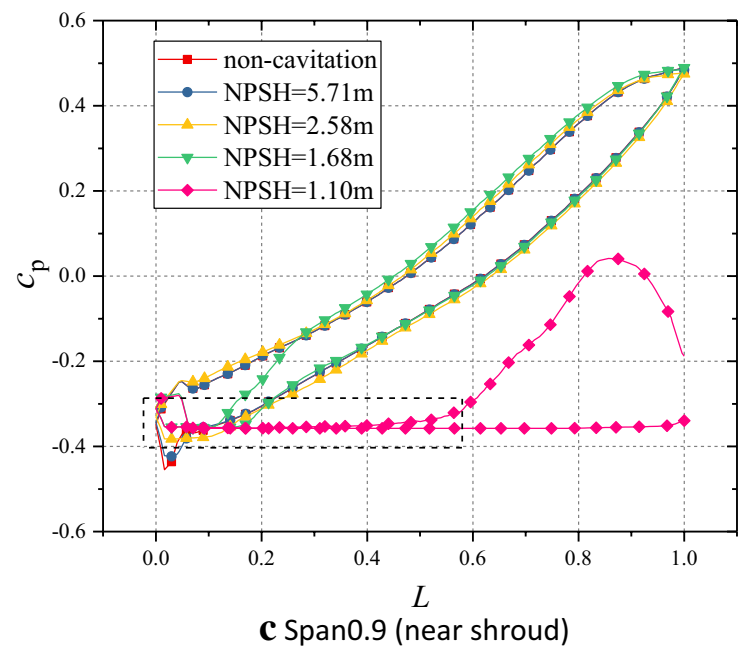

Figure 16 Blade loads curves under different cavitation status appears to decrease significantly because of the effect of the cavitating bubbles. 2) From the stress distribution of the blade from the inlet to the outlet before the super-cavitation state, it is observed that the stress first increases and then decreases and, finally, increases again. It is also observed that the high stress is produced in the middle and the outlet of blade, and the maximum value of the stress on the pressure side of the blade decreases from $56 \mathrm{MPa}$ to $41 \mathrm{MPa}$ with the transition from the non-cavitation state to the severe cavitation state. However, for the suction side of the blade, the maximum value of stress decreases from $225 \mathrm{MPa}$ to $166 \mathrm{MPa}$. In the super-cavitation state, the flow channels of the impeller are approximately occupied by bubbles, which weakens the fluid force on the blade surface, so the blade surface stress decreases significantly. However, the stress level remains stable at a low level from the inlet to the outlet of the blade. 3) In the analysis of the stress distribution trend, the stress on the suction side is typically greater than on the pressure side. Because the blade surface of the pressure side is in the tensile state under pressure loads and the force acting on blade surface is relatively uniform, it does not result in a local stress concentration problem. However, on the blade suction side, where the blade surface is in a compressed state, specifically at the end of the blade, a large deformation is produced with high pressure loads. Therefore, a greater stress concentration will be observed in this area.

\subsection{Natural Frequencies of Rotor System under Different Cavitation Conditions}

Table 3 presents the natural frequency variations of the centrifugal pump rotor system under different cavitation conditions. It can be seen that the natural frequencies of the centrifugal pump tend to decrease slightly with development of cavitation. For instance, the decrease of the first order of the natural frequency is $0.95 \mathrm{~Hz}$ from the non-cavitation state to the super-cavitation state. In addition, it can be seen that the first order natural frequency under the super-cavitation condition is the lowest with a value of $135.84 \mathrm{~Hz}$, which is greater than the rotor frequency of $48.3 \mathrm{~Hz}$, but smaller than the blade passing frequency of $241.7 \mathrm{~Hz}$. Therefore, the system could experience a cycle of the fluid excitation resonance phenomenon during operation, which should be prevented in the design and installation processes to avoid damage to the system. In addition, as for the second order to the fifth order of the natural frequency, the overall variation of the natural frequency under different cavitation conditions is less than $0.3 \mathrm{~Hz}$. Furthermore, it is also important to 


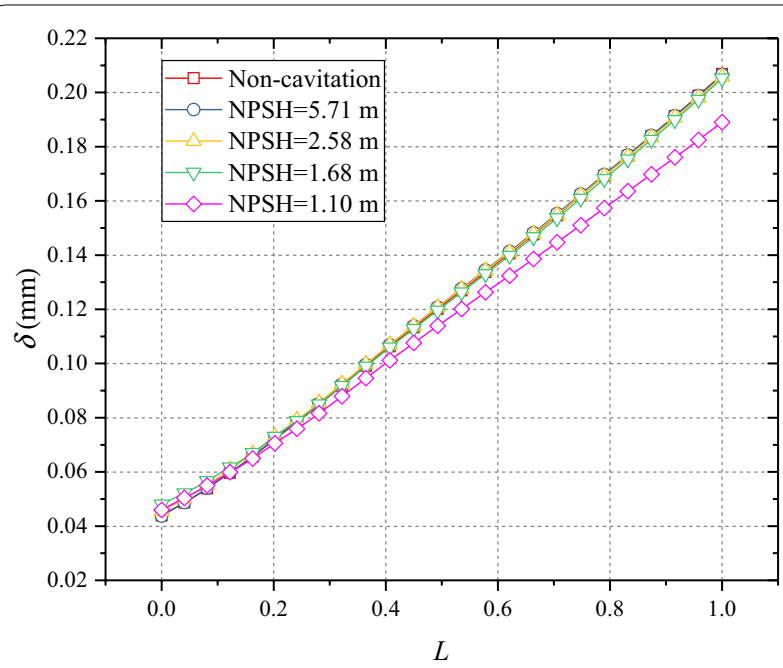

a $\mathrm{PH}$

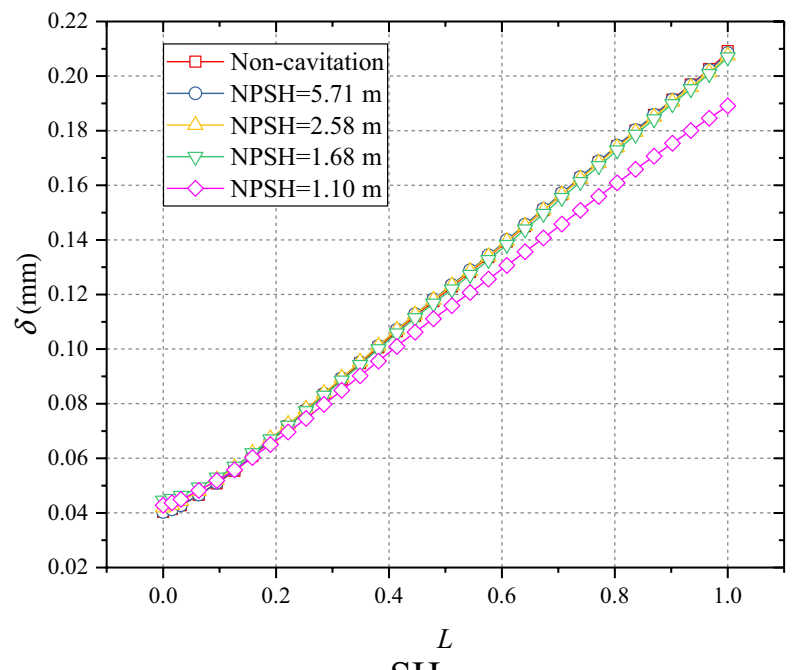

c $\mathrm{SH}$

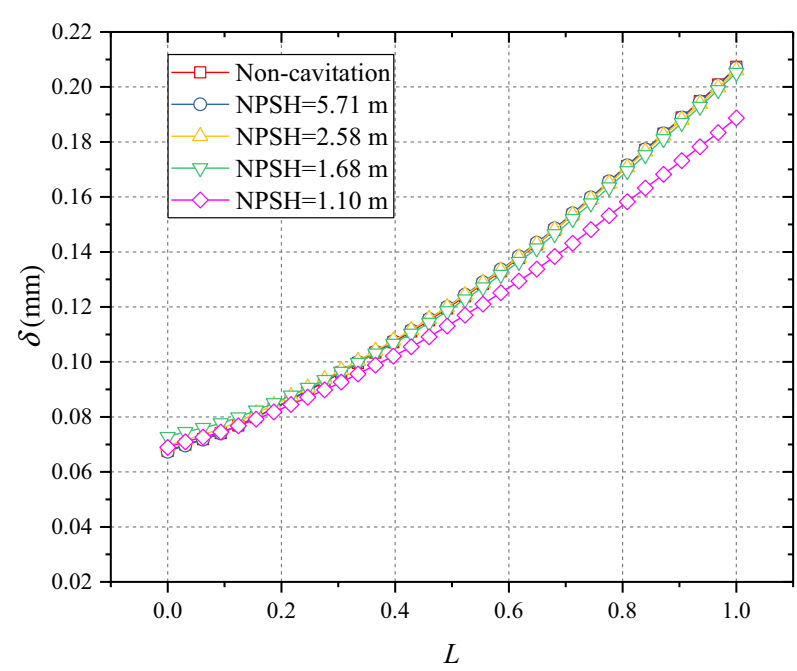

b PS

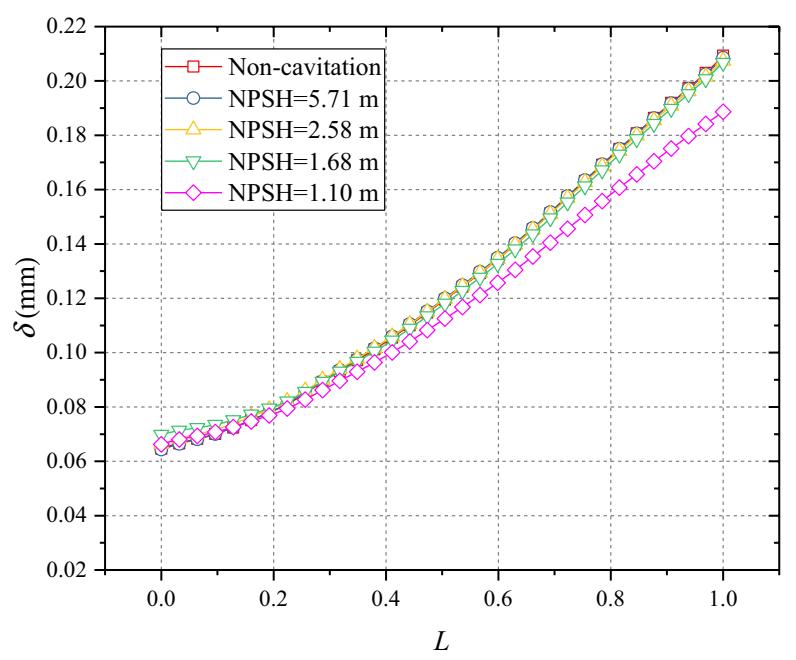

d SS

Figure 17 Blade deformation curves under different cavitation status

show that cavitation has little effect on the sixth order of the natural frequency. Figure 19 shows the six modes of the centrifugal pump in a super-cavitation state, and as the other cavitation states have a similar result. From the vibration mode point of view, the first three modes are bending deformation, and the fourth to sixth modes are torsional deformation. The mode results provide good data on how to improve the rotor instability, because each eigen mode is associated with a specific damping. As damping is proportional to the vibration velocity, if a bearing is located at or close to an amplitude maximum, rotor damping will be increased. Otherwise, it does not contribute to the damping.

\section{Conclusions}

(1) In this study, an FSI-based numerical method was used to investigate the effects of cavitation on the flow and structural behaviors in a centrifugal pump. Comparisons between simulation and experiment results were conducted for different flow rates under cavitation conditions. The results were in good agreement and were validated. The different cavitation states, from non-cavitation to super-cavitation, were mapped successfully, and the cavitating bubbles grew and expanded gradually as cavitation developed. 


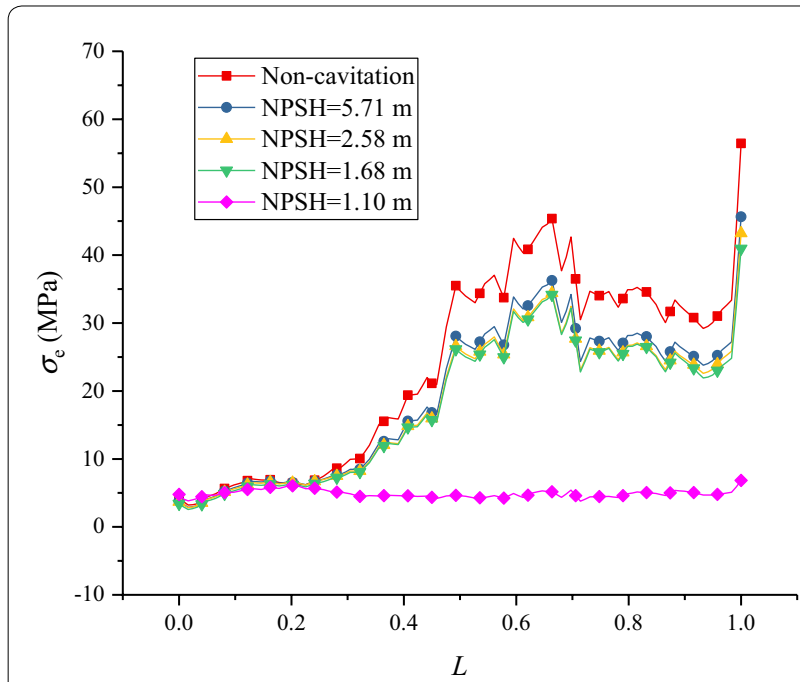

a $\mathrm{PH}$

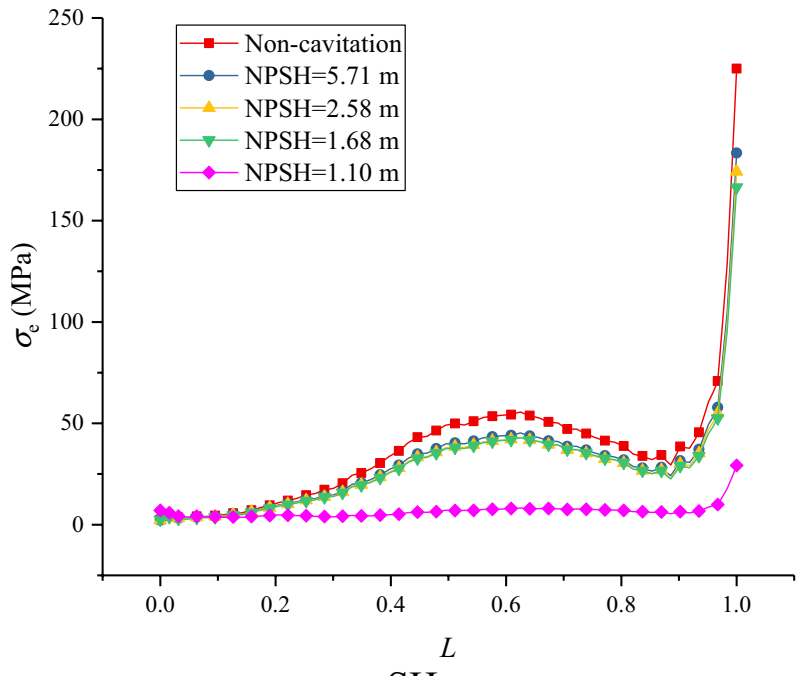

c $\mathrm{SH}$

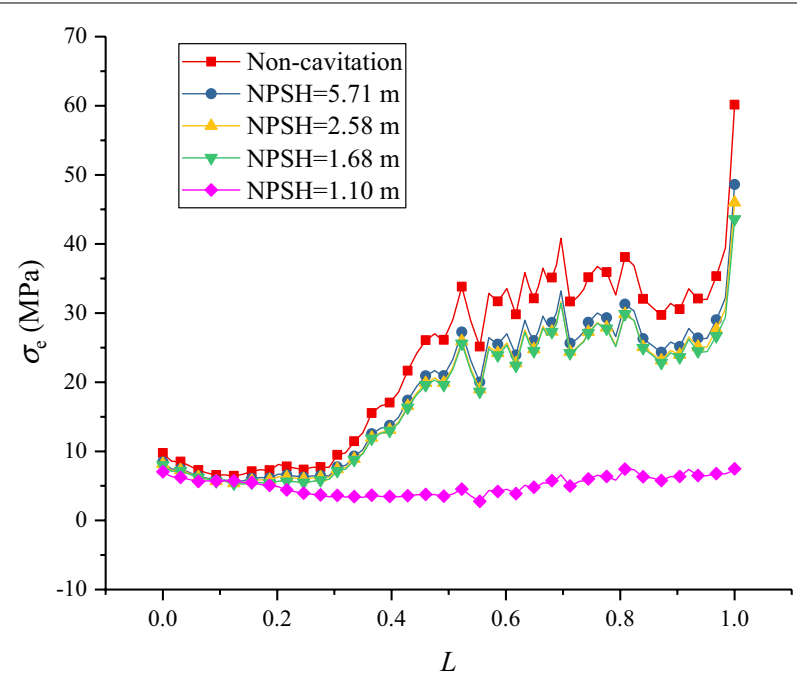

b PS

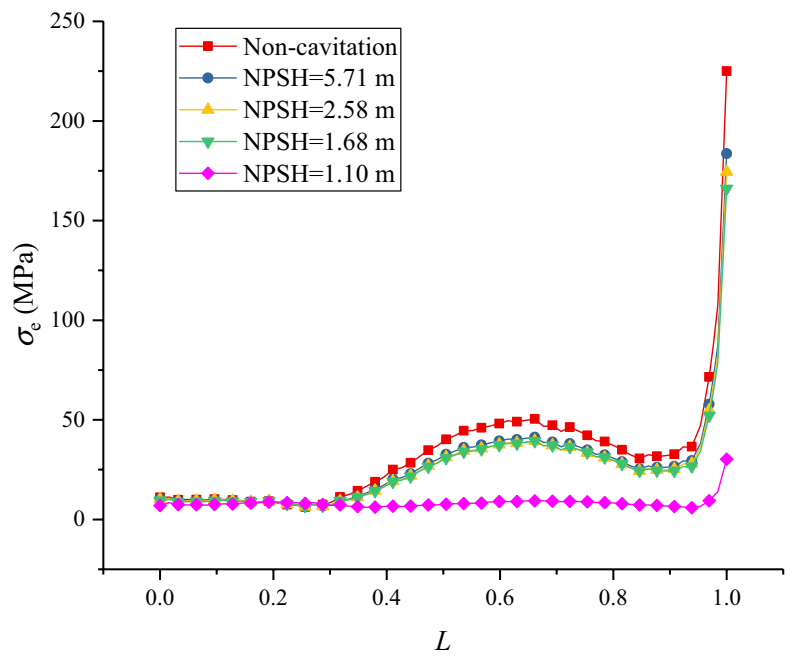

d SS

Figure 18 Blade stress curves under different cavitation status

Table 3 Natural frequencies of rotor system under different cavitation conditions

\begin{tabular}{|c|c|c|c|c|c|}
\hline Mode & Non-cavitation $(\mathrm{Hz})$ & $\mathrm{NPSH}=5.71 \mathrm{~m}(\mathrm{~Hz})$ & $\mathrm{NPSH}=2.58 \mathrm{~m}(\mathrm{~Hz})$ & $\mathrm{NPSH}=1.68 \mathrm{~m}(\mathrm{~Hz})$ & $\begin{array}{l}\mathrm{NPSH}=1.10 \mathrm{~m} \\
(\mathrm{~Hz})\end{array}$ \\
\hline 1 & 136.79 & 136.48 & 136.41 & 136.35 & 135.84 \\
\hline 2 & 156.98 & 156.92 & 156.91 & 156.89 & 156.70 \\
\hline 3 & 156.99 & 156.93 & 156.91 & 156.90 & 156.71 \\
\hline 4 & 779.66 & 779.60 & 779.59 & 779.57 & 779.44 \\
\hline 5 & 779.84 & 779.78 & 779.77 & 779.75 & 779.62 \\
\hline 6 & 1441.4 & 1441.4 & 1441.4 & 1441.4 & 1441.4 \\
\hline
\end{tabular}

(2) For a blade, the amplitude of the pressure fluctuation close to the suction side was significantly smaller than that on the pressure side at the same radius. The unsteady fluid flow was isolated by the bubbles that kept the pressure inside the bubble zone constant, and the amplitude was not dis- 
a

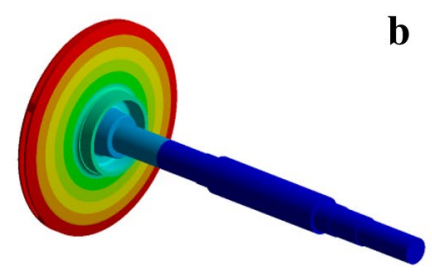

b

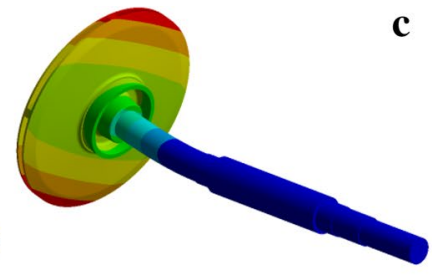

c

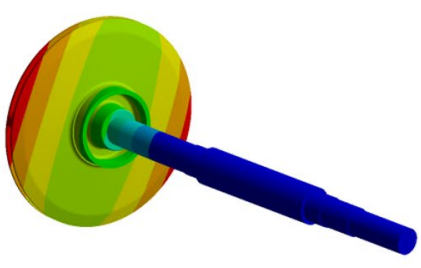

d
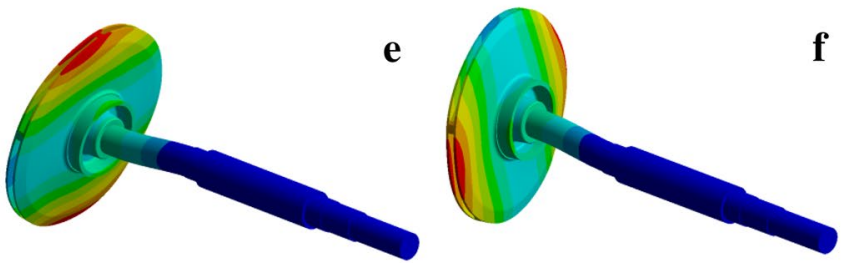

$\mathbf{f}$

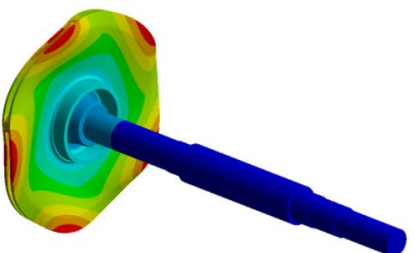

Figure 19 Mode analysis of rotor system: (a) mode 1; (b) mode 2; (c) mode 3; (d) mode 4; (e) mode 5; and (f) mode 6

cernible. However, for the zone with no bubbles, the large vortex and strong flow separation were strengthened by the cavitating flow, and the pressure fluctuation amplitude increased because of the blockage and collapse of bubbles. Furthermore, the amplitudes of the pressure oscillations in a volute were affected by bubble size and the volute tongue. Significant interaction between the blades and the tongue, together with strong cavitation flow, increased the amplitude of the pressure fluctuation.

(3) The radial force was significantly affected by the cavitation, with a maximum value of $54.4 \mathrm{~N}$ in the super-cavitation state. In the transition progress from the non-cavitation state to the severe cavitation state, the blade deformation remained approximately constant, with a maximum value of approximately $0.21 \mathrm{~mm}$. When the impeller entered the super-cavitation state, the deformation decreased, with a maximum value of $0.19 \mathrm{~mm}$. The stress of the blade and natural frequencies of the rotor system gradually decreased as cavitation developed. Specifically, in the super-cavitation state, the stress decreased significantly and remained approximately stable and small from the blade inlet to the outlet.

\section{Authors' Contributions}

DW carried out the experiments, participated in the data post processing and drafted the manuscript; YR assisted with CFD calculation; JM provided guidance on the experiment design and the data analysis methods; $Y G$ and LJ participated in the test and data post processing. All authors read and approved the final manuscript.

\section{Author Details}

${ }^{1}$ College of Mechanical Engineering, Zhejiang University of Technology, Hangzhou 310014, China. ${ }^{2}$ Zhijiang College, Zhejiang University of Technology, Hangzhou 310024, China.

\section{Authors' Information}

Denghao Wu, born in 1985, is currently an associate professor at Zhijiang College and Postdoctor at College of Mechanical Engineering, Zhejiang University of Technology, China. He received his PhD degree from Jiangsu University, China, in 2013. His research interests include cavitation and centrifugal pump optimization design.

Yun Ren, born in 1985, is currently a lecturer at Zhijiang College, Zhejiang University of Technology, China. She received her PhD degree from Jiangsu University, China, in 2014. Her research interests include computational fluid dynamics on centrifugal pump.

Jiegang Mou, born in 1963, is currently a professor at College of Mechanical Engineering, Zhejiang University of Technology, China. He received his PhD degree from Zhejiang University, China, in 2005.

Yunqing Gu, born in 1982, is currently a lecturer at College of Mechanical Engineering, Zhejiang University of Technology, China. He received his PhD degree from Harbin Engineering University, China, in 2013.

Lanfang Jiang, born in 1979, is currently an associate professor at Zhijiang College, Zhejiang University of Technology, China. She received her PhD degree from Zhejiang University of Technology, China, in 2009.

\section{Competing Interests}

The authors declare that they have no competing interests.

\section{Funding}

Supported by National Natural Science Foundation of China (Grant Nos. 51609212, 51606167), China Postdoctoral Science Foundation (Grant No. 2016M590546), and Zhejiang Provincial Natural Science Foundation (Grant No. 2016C31043).

\section{Publisher's Note}

Springer Nature remains neutral with regard to jurisdictional claims in published maps and institutional affiliations.

Received: 14 September 2017 Accepted: 18 February 2019

Published online: 28 February 2019

\section{References}

[1] CE Brennen. Cavitation and bubble dynamics. Oxford: Oxford University Press, 1995.

[2] S Duplaa, O Coutier-Delgosha, A Dazin, et al. Experimental study of a cavitating centrifugal pump during fast startups. Journal of Fluids Engineering, 2010, 132(2): 365-368. 
[3] J Friedrichs, G Kosyna. Unsteady PIV flow field analysis of a centrifugal pump impeller under rotating cavitation. Proceedings of the 5 th International Symposium on Cavitation, Osaka, Japan, November 2003.

[4] J Friedrichs, G Kosyna. Rotating cavitation in a centrifugal pump impeller of low specific speed. Journal of Fluids Engineering, 2002, 124(2): 356-362.

[5] YWang, H L Liu, D X Liu, et al. Application of the two-phase three-component computational model to predict cavitating flow in a centrifugal pump and its validation. Computers and Fluids, 2016, 133: 142-150.

[6] W Hassan, S Barre, S Legoupil. Study of the behavior of vapor fraction in a turbopump inducer using an X-ray measurement technique. Experiments in Fluids, 2014, 55(5): 1726

[7] Y Xu, L Tan, Y Liu, et al. Pressure fluctuation and flow pattern of a mixedflow pump with different blade tip clearances under cavitation condition. Advances in Mechanical Engineering, 2017, 9(4): 1-12.

[8] Y C Wang, L Tan, B S Zhu, et al. Numerical investigation of influence of inlet guide vanes on unsteady flow in a centrifugal pump. Proceedings of the Institution of Mechanical Engineers, Part C: Journal of Mechanical Engineering Science, 2015, 229(18): 3405-3416.

[9] R Tao, R F Xiao, F J Wang, et al. Cavitation behavior study in the pump mode of a reversible pumpturbine. Renewable Energy, 2018, 125: 655-667.

[10] O Coutier-Delgosha, R Fortes-Patella, J Reboud, et al. Experimental and numerical studies in a centrifugal pump with two-dimensional curved blades in cavitating condition. Journal of Fluids Engineering, 2003, 125(6): 970-978.

[11] L Meng, M He, L Zhou, et al. Influence of impeller-tongue interaction on the unsteady cavitation behavior in a centrifugal pump. Engineering Computations, 2015, 33(1): 171-183.

[12] J X Lu, S Q Yuan, S Parameswaran, et al. Investigation on the vibration and flow instabilities induced by cavitation in a centrifugal pump. Advances in Mechanical Engineering, 2017, 9(4): 1-11.
[13] J X Lu, S Q Yuan, S Parameswaran, et al. The characteristics investigation under the unsteady cavitation condition in a centrifugal pump. Journal of Mechanical Science and Technology, 2017, 31(3): 1213-1222.

[14] L d'Agostino, F d'Auria, C E Brennen, et al. A three-dimensional analysis of rotordynamic forces on whirling and cavitating helical inducers. Journal of Fluids Engineering, 1998, 120(4): 698-704.

[15] Q Fu, F Zhang, R S Zhu, et al. A systematic investigation on flow characteristics of impeller passage in a nuclear centrifugal pump under cavitation state. Annals of Nuclear Energy, 2016, 97: 190-197.

[16] D Valentini, G Pace, A Pasini, et al. Fluid-induced rotordynamic forces on a whirling centrifugal pump. European Journal of Mechanics B-Fluids, 2017, 61(2): 336-345

[17] R L Campbell, E G Paterson. Fluid-structure interaction analysis of flexible turbomachinery. Journal of Fluids and Structures, 2011, 27(8): 1376-1391.

[18] C D Peters, S J Spuy, D N Els, et al. Aerodynamic damping of an oscillating fan blade: Mesh-based and meshless fluid structure interaction analysis. Journal of Fluids and Structures, 2018, 82: 173-197.

[19] M Song, J Ma, Y Huang. Fluid-structure interaction analysis of ship-ship collisions. Marine Structures, 2017, 55: 121-136.

[20] R Messahel, C Regan, M Souli. Numerical investigation of homogeneous equilibrium model and fluid-structure interaction for multiphase water flows in pipes. International Journal of Multiphase Flow, 2018, 98: 56-66.

[21] F Benra, H Dohmen, J Pei, et al. A comparison of one-way and two-way coupling methods for numerical analysis of fluid-structure interactions. Journal of Applied Mathematics, 2011, 2011: 36-47.

[22] J Pei, H Dohmen, S Q Yuan, et al. Investigation of unsteady flow-induced impeller oscillations of a single-blade pump under off-design conditions. Journal of Fluids and Structures, 2012, 35: 89-104.

[23] V Yakhot, S Orszag. Renormalization group analysis of turbulence. Physical Review Letters, 1986, 57(14): 1722-1724.

\section{Submit your manuscript to a SpringerOpen ${ }^{\odot}$ journal and benefit from:}

- Convenient online submission

- Rigorous peer review

- Open access: articles freely available online

- High visibility within the field

- Retaining the copyright to your article

Submit your next manuscript at springeropen.com 